\title{
Zygomatic bone implants in prosthetic rehabilitation - A review
}

\author{
Nandeeshwar DB' ${ }^{1}$ Neha Arora ${ }^{2}$
}

Professor and Head' ${ }^{1}$, Post Graduate Student ${ }^{2}$

Department of Prosthodontics and Crown and Bridge, Bapuji Dental College \& Hospital, Davangere

\begin{abstract}
:
Dental implants are the new era in the field of dentistry providing the new opportunities to the clinicians to manage their patients with missing teeth. The procedure is more technique sensitive in maxilla than mandible. The scenario becomes even more challenging with severely resorbed maxillary arches. The idea of zygomatic bone implants put forward the new approach to manage such patients. The purpose of the present article is to describe the concept of zygomatic implantology with emphasis on case selection and clinical outcomes based on the literature.
\end{abstract}

Keywords: Zygomatic fixture, Bone graft, Immediate/ Early loading

\section{Introduction:}

Replacement of missing teeth is one of the common complaint for which the patient visits the dentist. There are basically three techniques to manage these conditions in mouth i.e. removable denture, tooth supported fixed denture and implant supported fixed dentures. Every technique has its own advantages and disadvantages. Implant supported fixed treatment is preferred by the patients because of favourable outcomes. In many patients conventional implant treatment cannot be performed in the edentulous maxilla because of extensive bone resorption and the presence of larger maxillary sinuses, leading to inadequate amounts of bone tissue for anchorage of the implants. The treatment option for these patients has often been bone-augmentation procedures in order to increase the volume of load-bearing bone. Traditionally, the atrophic maxilla has been treated with large bone grafts from the iliac crest. This procedure is more invasive and requires general anesthesia. It can be argued that bone-augmentation procedures are resource demanding, take a long time and may present risk for morbidity of the donor site of the bone graft. It is also obvious that failure rates are higher in grafted than in non grafted maxillae.

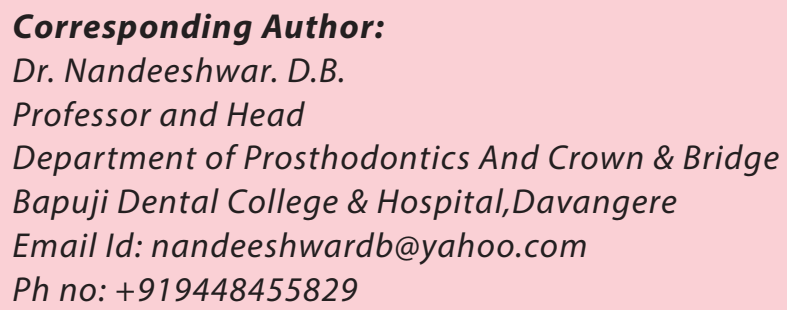

One alternative to bone grafting that has been considered in the atrophied maxilla is the use of the zygomatic implants. ${ }^{1}$ The zygomatic fixture is the result of development of reconstructive techniques for prosthetic rehabilitation of patients with extensive defects of the maxilla caused by tumor resection, trauma and congenital defects. The bone of the zygomatic arch was used for anchorage of a long fixture, which, together with ordinary fixtures, could be used as an anchor for epistheses, prostheses and obturators. The technique has enabled sufficient rehabilitation of these patients, with restored function and improved esthetics as a result, and thus has given many patients back a normal social life. The purpose of the present article is to describe the concept of zygomatic implantology with emphasis on case selection and clinical outcomes based on the literature.

\section{Case selection for zygomatic implant (Figure 1 and 2)}

The zygomatic bone has a pyramidal shape and contains dense cortical and trabecular bone. ${ }^{2}$ According to a cadaver study, the mean length of available bone in this region is about $14 \mathrm{~mm} .{ }^{2}$ Firstly, zygomatic fixtures can be used in patients with severely resorbed edentulous maxillary arches posterior to canine region (i.e. $<4 \mathrm{~mm}$ bone height distal to the canines), but with sufficient amounts of bone in the anterior region. Together with conventional implants in the anterior region of maxilla, the zygomatic fixture offers anchorage for a fixed bridge using less invasive surgery compared with bone-augmentation procedures. Secondly, for patients 
with smaller bone volumes in the anterior part of the maxilla, the zygomatic implant can be used in conjunction with a bone-augmentation procedure of the anterior segment. In this way, fewer bone grafts are needed for the augmentation procedure. Zygomatic implants are also indicated when contraindications exist for harvesting of the iliac crest bone graft. The main advantage with the technique is that it can be performed as an outpatient procedure under local anesthesia and conscious sedation. However, for better comfort for the patient, the routine procedure is usually performed under general anesthesia

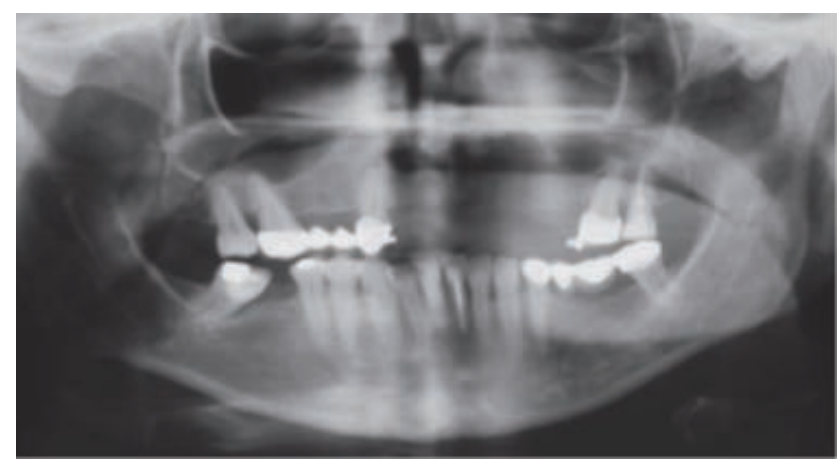

Figure 1: Pre-operative OPG of a case of partial edentulism treated with Zygomatic implant. $^{3}$

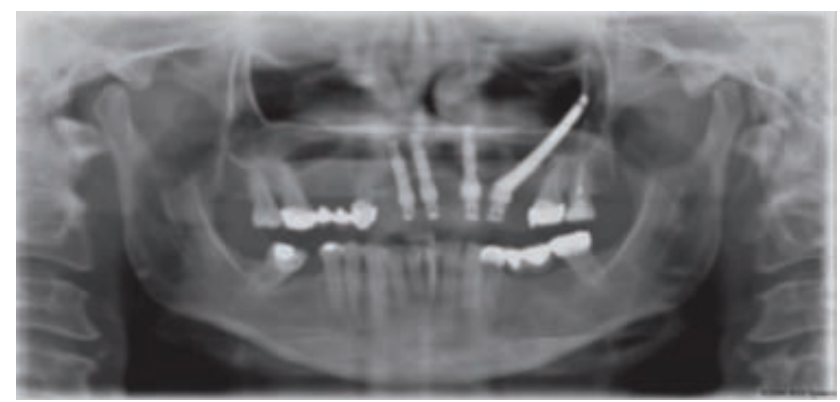

Figure 2: Post-operative OPG of a case of partial edentulism treated with Zygomatic implant. $^{3}$

The computed tomographic imaging can be used to evaluate the zygomatic implant site for the amount of bone in the zygomatic arch and in the residual alveolar crest. The angulation, expected emergence site and the relationship of the implant body to the maxillary sinus and lateral wall should be evaluated. The emergence of the head of the implant in relation to the alveolar crest, typically in the palatal aspect of the second premolar region, is therefore dependent on the spatial relationship between the zygomatic bone, the maxillary sinus and the alveolar crest. A new technique, including extrasinus passage of the implant, has been evaluated with promising results. This facilitates an optimal positioning of the zygomatic fixture head in relation to the alveolar crest and the occlusal table of the prosthetic construction. Any pathosis of the maxillary sinus should preferably be treated prior to installation of the zygomatic fixture.

\section{Zygomatic Implant design and clinical procedure}

The original zygomatic fixture is a self-tapping titanium implant with a machined surface and is available in lengths from 30 to $52.5 \mathrm{~mm}$. The threaded apical part has a diameter of $4 \mathrm{~mm}$ and the crestal part has a diameter of $4.5 \mathrm{~mm}$. The implant head has an angulation of $45^{\circ}$ and an inner thread for connection of Branemark System abutments. Zygomatic fixtures are currently commercially available from at least three different companies that offer implants with an oxidized rough surface, a smooth mid implant body, a wider neck at the alveolar crest and a $55^{\circ}$ angulation of the implant head.

\section{Step 1: Surgical technique (Figure 3 and 4)}

Surgery is usually carried out under general anesthesia. In those patients, local anesthesia is infiltrated in the maxillary vestibulum, in the area of the zygomatic bone and $1 \mathrm{~cm}$ palatal to the bone crest. The area is exposed via a midcrestal incision and vertical releasing incisions along the posterior part of the infrazygomatic crest and anterior to the surgical site. The vertical ridge/anterior border of the zygomatic arch is identified. A second landmark is the lateral orbital border. Mucoperiosteal flap elevation and exposure of the central/posterior part of the zygomatic complex, avoiding interference with the orbita, the lateral wall of the maxillary sinus and the alveolar crest, are carried out. A retractor is positioned for visibility and to protect the soft tissues. An indicator is used to determine the drilling direction and the starting point at the crest, usually in the second premolar / first molar region. A bone window, some $10 \mathrm{~mm}$ wide, is created at the lateral aspect of the maxillary sinus following the desired path of the zygomatic implant from the sinus floor to the top of the sinus cavity. The sinus membrane is carefully dissected free from the sinus walls into the sinus cavity. 
A series of drills is used to penetrate the alveolar process and the zygomatic bone. The estimated length of the zygomatic implant is selected using a depth gauge. The self-tapping zygomatic implant is placed with the aid of a motor or manually using a fixture mount. Care should be taken not to enlarge the palatal hole at the rest during insertion, which is especially important in cases of thin alveolar/basal bone. If needed, bone particles harvested locally can be packed around the zygomaticus implant. A cover screw is placed on the implant and the mucoperiosteal flap is closed. Abutment connection is usually made after a healing period of 6 months following the procedures for the Branemark System using standard or straight / angulated multiunit Branemark abutments.

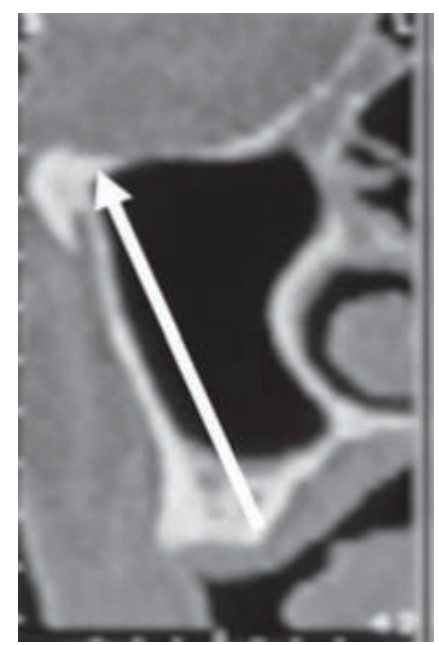

Figure 3: Tomographic section showing the intrasinus path of the zygomatic implant (arrow). ${ }^{13}$

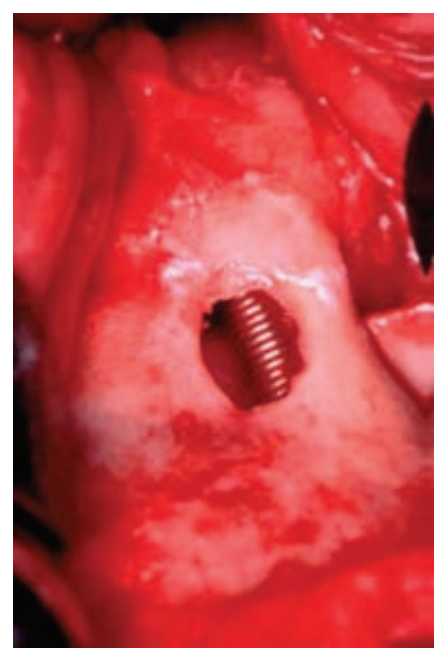

Figure 4: Clinical photograph showing a lateral window of the maxillary sinus for visual control of implant insertion. ${ }^{13}$

\section{Step 2: Prosthetic procedure (Figure 5 and 6)}

The prosthetic procedure follows conventional protocols for cemented or screw-retained implant-supported dental bridges. As the emergence of the zygomatic implant is often some 10-15 mm medial to the ridge, the bridge should be designed to enable proper oral hygiene in the area.

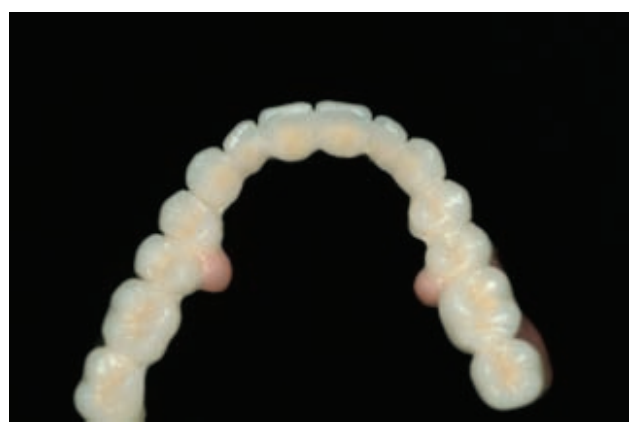

Figure 5: Clinical photograph showing the final prosthetic construction. ${ }^{13}$

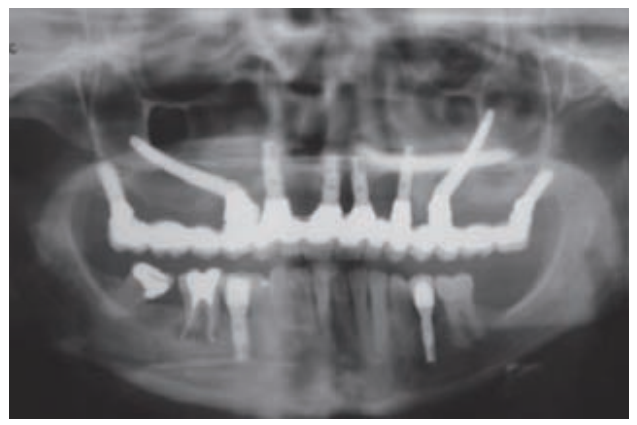

Figure 6: OPG of the completed procedure showing two zygomatic implants and six standard implants, where two are in the tuberosity area and four are in the premaxilla. ${ }^{11}$

\section{Clinical outcome of using the zygomatic implant}

In a literature review of 18 studies presenting clinical outcomes with the zygomatic fixture were found (Table 1). The publications included 537 patients and 1056 zygomatic implants with a follow-up of 6 months- 12 years. A total of 18 implants were reported as failures, giving an overall survival rate of $98.29 \%$. However, it should be noted that some studies in part cover the same patient groups and therefore the true numbers of unique patients and implants are not known in detail. Nevertheless, the data show that the zygomatic implant technique is highly predictable and results in good clinical outcomes. 
Table 1: Clinical Outcome of the Zygomatic Implants ${ }^{1,4-20}$

\begin{tabular}{|c|c|c|c|c|}
\hline Study & $\begin{array}{c}\text { No. of } \\
\text { Patients }\end{array}$ & $\begin{array}{c}\text { Time period } \\
\text { of Follow up }\end{array}$ & $\begin{array}{c}\text { Total No. of } \\
\text { Zygomatic } \\
\text { Implants }\end{array}$ & $\begin{array}{c}\text { Total no. of } \\
\text { Faliures }\end{array}$ \\
\hline Branemark et al. $^{1}$ & 81 & $1-10$ & 164 & 4 \\
\hline Becktor et al. $^{4}$ & 16 & $1-6$ years & 31 & 3 \\
\hline Bedrossian et al. $^{5}$ & 22 & 34 months & 44 & 0 \\
\hline Malevez et al. $^{6}$ & 55 & $0 .-4$ years & 103 & 0 \\
\hline Hirsch et al. $^{7}$ & 66 & 1 year & 124 & 3 \\
\hline Branemark et al. $^{\mathbf{}}$ & 28 & $5-10$ years & 52 & 3 \\
\hline Parel et al. $^{{ }^{7}}$ & 27 & $1-12$ & 65 & 0 \\
\hline Vrielinck et al. $^{10}$ & 29 & $<2$ years & 46 & 3 \\
\hline Boyes-Varley et al. $^{11}$ & 45 & $6-30$ months & 77 & 0 \\
\hline Penarrocha et al. $^{12}$ & 5 & $1-1.5$ years & 10 & 0 \\
\hline Farzad et al. $^{13}$ & 11 & $1.5-4$ years & 22 & 0 \\
\hline Ahlgren et al. $^{14}$ & 13 & $1-4$ years & 25 & 0 \\
\hline Aparicio et al. $^{15}$ & 69 & $0.5-5$ years & 131 & 0 \\
\hline Bedrossian et al. $^{16}$ & 14 & $>12$ months & 28 & 0 \\
\hline Chow et al. $^{17}$ & 5 & 10 months & 10 & 0 \\
\hline Duarte et al. $^{18}$ & 12 & 30 months & 48 & 2 \\
\hline Penarrocha et al. $^{19}$ & 21 & $12-45$ months & 40 & 0 \\
\hline Davo et al. $^{20}$ & 18 & $6-29$ months & 36 & 0 \\
\hline
\end{tabular}

\section{Complications associated with the procedure}

The zygomatic implant prosthesis system is complex from the biologic point of view as a result of the interfaces towards different tissues such as bone, oral mucosa and sinus mucosa. The passage of the fixture itself through the sinus cavity does not seem to provoke any severe negative soft tissue reactions, as evaluated by sinuscopy of 14 patients. ${ }^{3}$ However, few clinical follow-up studies on zygomatic implants report on soft tissue complications intraorally or in the maxillary sinus. As mentioned in the previous paragraph, Becktor et al. had to remove three of 31 implants because of recurrent sinusitis, in spite of the implants being clinically stable. ${ }^{4}$ They proposed two explanations for their problems: either the internal threaded abutment screw chamber of the zygomatic implant created a communication from the oral cavity into the maxillary sinus, which may have resulted in sinusitis, or a lack of osseointegration occurred at the marginal level in the palatal area, which resulted in transversal mobility of the zygomatic implant and a pump effect during function. ${ }^{4}$ Other researchers have reported sinusitis to occur in $2.3-13.6 \%$ of the sinuses treated. ${ }^{5-8}$ Intra-oral infections seem to occur at a similar rate (i.e. from 3.8 to $6.5 \%),{ }^{7,8}$ except in the study carried out by Becktor et al. (29\%). ${ }^{4}$ Another study reported that nine of 20 zygomatic implants were associated with periimplant bleeding and increased probing depths, possibly caused by difficulties in implementing appropriate hygiene because of the positioning of the zygomatic implant head and abutment, and the design of the prosthesis. ${ }^{3}$ Thus, the risk of soft tissue problems and sinusitis should not be underestimated.

\section{Recent developments of the zygomatic fixture technique}

\section{Placement in local anaesthesia}

One further simplification of the technique is the use of local anesthesia and oral or intravenous sedation. This procedure is recommended if the surgeon is experienced and the procedure takes less than 1.5 hours. ${ }^{3}$ The authors experienced that the procedure is well tolerated by the patient and that the surgery is facilitated by working on a conscious patient.

\section{Immediate loading}

Numerous clinical follow-up studies have reported good results with immediate / early loading protocols of maxillary prosthesis in 12 months follow up of their patients. ${ }^{2}$ In pioneering studies, the good results can also be attributed to the fact that the implants can be placed in an arch form, which counteracts bending forces. ${ }^{5,6,7}$

\section{Extrasinus placement}

One drawback with the zygomatic implant technique is the palatal emergence of the implant head, which is often the case because of the desire to maintain the implant body within the boundaries of the maxillary sinus. This commonly results in a bulky dental bridge at the palatal aspect, which sometimes leads to discomfort and problems with oral hygiene. Zygomatic implant placement with an intrasinus path may even be impossible in patients with pronounced buccal concavities at the lateral aspect of the maxillary sinus. Therefore, an extrasinusal approach to placement of zygomatic implants has been developed to obtain the implant head emergence at or near the top of the residual alveolar crest, usually in the second premolar/first molar regions. Moreover, the implant body should preferably engage the lateral bone wall of the maxillary sinus while entering the zygomatic bone. The implant site is prepared without making an opening to the maxillary sinus and otherwise follows the standard drilling steps for zygomatic implants. 
This leading author has experience with the extrasinus technique in 20 patients with pronounced buccal concavities in whom 36 zygomatic implants and 104 conventional implants were placed with zygomatic implants using sinuscopy and found no signs of adverse reactions. ${ }^{3}$

\section{Conclusion}

To summarize, zygomatic implants are very useful in the prosthetic rehabilitation of the severely resorbed maxilla, regardless of whether it is totally edentulous or partially edentulous individuals. A review of literature showed that good clinical outcome can be achieved. However, in comparison with bone grafting procedures, the technique is less invasive and complicated and has a lower risk of morbidity because of the fact that harvesting of bone graft is usually not needed. In patients with insufficient bone volume in the anterior region, bone grafting may be required in order to enable placement of conventional implants. However, fewer bone grafts are needed because the posterior maxilla does not need any augmentation procedure. Bone grafting should still be regarded as one option for reconstruction of the severely resorbed maxilla and is necessary in patients where reconstruction of the facial morphology and correction of the intermaxillary relation is desired.

\section{References}

1. Branemark PI, Grondahl K, Ohrnell LO, Nilsson P, Petruson B, Svensson B, Engstrand P, Nannmark U. Zygoma fixture in the management of advanced atrophy of the maxilla: Technique and long-term results. Scand J Plast Reconstr Surg Hand Surg 2004: 38: 70-85.

2. Olsson M, Urde G, Andersen JB, Sennerby L. Early loading of maxillary fixed cross-arch dental prostheses supported by six or eight oxidized titanium implants: results after 1 year of loading, case series. Clin Implant Dent Relat Res 2003: 5(Suppl. 1): 81-87.

3. Carlos Aparicio, Wafaa Ouazzani, Naoki Hatano. The use of zygomatic implants for prosthetic rehabilitation of the severely resorbed maxilla. Periodontology 2000 2008;47:162-71.

4. Becktor JP, Isaksson S, Abrahamsson P, Sennerby L. Evaluation of 31 zygomatic implants and 74 regular dental implants used in 16 patients for prosthetic reconstruction of the atrophic maxilla with cross-arch fixed bridges. Clin Implant Dent Relat Res 2005: 7: 159-165.

5. Bedrossian E, Stumpel L III, Beckely ML, Indresano T. The zygomatic implant: preliminary data on treatment of severely resorbed maxillae. A clinical report. Int J Oral Maxillofac Implants 2002: 17: 861-865.

6. Malevez C, Abarca M, Durdu F, Daelemans P. Clinical outcome of 103 consecutive zygomatic implants: a 6- 48 months follow-up study. Clin Oral Implants Res 2004: 15: 18-22.

7. Hirsch JM, Ohrnell LO, Henry PJ, Andreasson L, Branemark PI, Chiapasco M, Gynther G, Finne K, Higuchi KW, Isaksson S, Kahnberg KE, Malevez C, Neukam FW, Sevetz E, Urgell JP, Widmark G, Bolind P. A clinical evaluation of the Zygoma fixture: one year of follow-up at 16 clinics. J Oral Maxillofac Surg 2004: 62(9 Suppl. 2): 22-29.

8. Branemark PI, Grondahl K, Ohrnell LO, Nilsson P, Petruson B, Svensson B, Engstrand P, Nannmark U. Zygoma fixture in the management of advanced atrophy of the maxilla: technique and long-term results. Scand J Plast Reconstr Surg Hand Surg 2004: 38: 70-85.

9. Parel SM, Branemark PI, Ohrnell LO, Svensson B. Remote implant anchorage for the rehabilitation of maxillary defects. J Prosthet Dent 2001: 86: 377-381.

10.Vrielinck L, Politis C, Schepers S, Pauwels M, Naert I. Image- based planning and clinical validation of zygoma and pterygoid implant placement in patients with severe bone atrophy using customized drill guides. Preliminary results from a prospective clinical follow-up study. Int J Oral Maxillofac Surg 2003: 32: 7-14.

11.Boyes-Varley JG, Howes DG, Lownie JF, Blackbeard GA. Surgical modifications to the Branemark zygomaticus protocol in the treatment of nthe severely resorbed maxilla: a clinical report. Int J Oral Maxillofac Implants 2003: 18: 232-237.

12.Penarrocha M, Uribe R, Garcia B, Marti E. Zygomatic implants using the sinus slot technique: clinical report of a patient series. Int $\mathrm{J}$ Oral Maxillofac Implants 2005: 20: 788-792.

13.Farzad P, Andersson L, Gunnarsson S, Johansson B. Rehabilitation of severely resorbed maxillae with zygomatic implants: An evaluation of implant stability, tissue conditions, and patients_ opinion before and after treatment. Int $\mathrm{J}$ Oral Maxillofac Implants 2006: 21: 399-404.

14.Ahlgren F, Storksen K, Tornes K. A study of 25 zygomatic dental implants with 11 to 49 months follow-up after loading. Int $\mathrm{J}$ Oral Maxillofac Implants 2006: 21: 421-425.

15.Aparicio C, Branemark PI, Keller EE, Olive J. Reconstruction of the premaxilla with autogenous iliac bone in combination with osseointegrated implants. Int J Oral Maxillofac Implants 1993: 8: 61-67. 
16.Bedrossian E, Rangert B, Stumpel L, Indresano T. Immediate function with the zygomatic implant: a graftless solution for the patient with mild to advanced atrophy of the maxilla. Int J Oral Maxillofac Implants 2006: 21: 937- 942.

17.Chow J, Hui E, Lee PK, Li W. Zygomatic implants protocol for immediate occlusal loading: a preliminary report. J Oral Maxillofac Surg 2006: 64: 804-811.

18.Duarte LR, Filho HN, Francischone CE, Peredo LG, Branemark PI. The establishment of a protocol for the total rehabilitation of atrophic maxillae employing four zygomatic fixtures in an immediate loading system $-\mathrm{a}$ 30- month clinical and radiographic follow-up. Clin Implant Dent Relat Res 2007: 9: 186-196.
19.Penarrocha M, Garcia B, Marti E, Boronat A. Rehabilitation of severely atrophic maxillae with fixed implant-supported prostheses using zygomatic implants placed using the sinus slot technique: clinical report on a series of 21 patients. Int J Oral Maxillofac Implants 2007: 22: 645-650.

20.Davo C, Malevez C, Rojas J. Immediate function in the atrophic maxilla using zygoma implants: a preliminary study. J Prosthetic Dent 2007: 97: S44-S51.

How to cite this article:

Nandeeshwar DB, Neha A. Zygomatic bone implants in prosthetic rehabilitation - A review. CODS J Dent 2014;6;90-95

Source of support: Nil. Conflict of interest: None Declared. 